\title{
Model studies of long Josephson junction arrays coupled to a high-Q resonator
}

\author{
Filatrella, G.; Rotoli, G.; Grønbech-Jensen, N.; Parmentier, R. D.; Pedersen, Niels Falsig
}

Published in:

Journal of Applied Physics

Link to article, DOI:

$10.1063 / 1.352343$

Publication date:

1992

Document Version

Publisher's PDF, also known as Version of record

Link back to DTU Orbit

Citation (APA):

Filatrella, G., Rotoli, G., Grønbech-Jensen, N., Parmentier, R. D., \& Pedersen, N. F. (1992). Model studies of long Josephson junction arrays coupled to a high-Q resonator. Journal of Applied Physics, 72(7), 3179-3185. https://doi.org/10.1063/1.352343

\section{General rights}

Copyright and moral rights for the publications made accessible in the public portal are retained by the authors and/or other copyright owners and it is a condition of accessing publications that users recognise and abide by the legal requirements associated with these rights.

- Users may download and print one copy of any publication from the public portal for the purpose of private study or research.

- You may not further distribute the material or use it for any profit-making activity or commercial gain

- You may freely distribute the URL identifying the publication in the public portal 


\title{
Model studies of long Josephson junction arrays coupled to a high- $Q$ resonator
}

\author{
G. Filatrella and G. Rotoli \\ Department of Physics, University of Salerno, I-84081 Baronissi (SA), Italy \\ N. Grønbech-Jensen \\ Physics Laboratory I, The Technical University of Denmark, DK-2800 Lyngby, Denmark \\ R. D. Parmentier \\ Department of Physics, University of Salerno, I-84081 Baronissi (SA), Italy \\ N. F. Pedersen \\ Physics Laboratory I, The Technical University of Denmark, DK-2800 Lyngby, Denmark
}

(Received 4 February 1992; accepted for publication 8 June 1992)

\begin{abstract}
Series-biased arrays of long Josephson junction fluxon oscillators can be phase locked by mutual coupling to a high- $Q$, linear distributed resonator. A simplified model of such a device, consisting of junctions described by the particle-map perturbation theory approach which are capacitively coupled to a lumped, linear tank circuit, reproduce the essential experimental observations at a very low computational cost. A more sophisticated model, consisting of partial differential equation descriptions of the junctions, again mutually coupled to a linear tank, substantially confirm the predictions of the simplified model. In the particle-map model, the locking range in junction bias current increases linearly with the coupling capacitance; in the partial differential equation (p.d.c.) model, this holds up to a certain maximum value of the capacitance, after which a saturation of the locking range is observed. In both models, for a given spread of junction lengths, the existence of a minimum value of the capacitance for locking to a tank with a given resonant frequency is evidenced.
\end{abstract}

\section{INTRODUCTION}

Recent experimental works by Monaco et al. ${ }^{1}$ and by Davidson $e t a l^{2}$ have clearly demonstrated that long Josephson junction oscillators (so-called fluxon oscillators) in a series-biased array can be locked together at a very sharp frequency by means of a mutual coupling to a high- $Q$ linear resonant cavity. Such devices appear to offer considerable promise as local oscillators in the microwave or millimeter wave region, in particular because, as shown by Monaco et al., ${ }^{1}$ the power level of the emitted signal varies, in appropriate circumstances, with the square of the number of active junctions, reaching levels almost high enough ( $10 \mathrm{nW}$ in a $50 \Omega$ load $^{1}$ ) to be practically useful. In order to realize this promise, i.e., to optimize the desired performance features of experimental devices, detailed model studies are of obvious utility. The present work is intended as a step in this direction.

The experimental device of Monaco et al. ${ }^{1}$ consists of a series-biased array of overlap-geometry junctions located near one end of a $50 \Omega$ microstrip resonator which is one half-wavelength long at the operating frequency, $(\sim 10$ $\mathrm{GHz}$ ). The device of Davidson et al., ${ }^{2}$ instead, has the junction array embedded in the side electrodes of a coplanar slotline resonator whose half-wavelength frequency is $\sim 35 \mathrm{GHz}$. Monaco et al. ${ }^{1}$ convincingly demonstrated that the first-order behavior of their device can be described by a simple model in which the physical, distributed resonator is replaced by a lumped, linear tank circuit. In this model each junction is coupled to the tank through a capacitance which also comprises a part of the resonator, and the dynamics of the oscillating fluxon in each junction is described using the particle-map approach developed by Salerno et $a l^{3}$ to model the behavior of a single longjunction oscillator, biased on its first zero-field step, in the presence of an external microwave field. It seems reasonable to suppose that this simple model might also be applicable, at least as a rough approximation, to the device of Davidson et $a l^{2}$

The usefulness of the combined model, consisting of the particle-map description of propagating fluxons, together with a lumped resonator, derives from the fact that it provides quite reasonable approximate results at an extremely low computational cost; however, it certainly does represent a rather drastic simplification of the physical system. An obvious limitation of the model is that a lumped resonator oscillates at a single frequency, whereas a distributed resonator can also respond at harmonics of its fundamental frequency. Moreover, partial differential equation (p.d.e.) descriptions of fluxon dynamics reveal a number of features, ${ }^{4}$ in particular fluxon creation, that are not captured by the particle-map approach. ${ }^{3}$ Nonetheless, the overall computational economy offered by the simplified model amply justifies its use, in particular, for studies involving preliminary explorations of parameter space.

It is just this type of application that we address in the present article, viz., the influence on the system dynamics of the tank capacitance parameter; in particular, we study the dependence of the locking range in bias current on this parameter, and we demonstrate that there exists a minimum value of capacitance for locking. In order to further the credibility of the simplified model, but also to highlight 


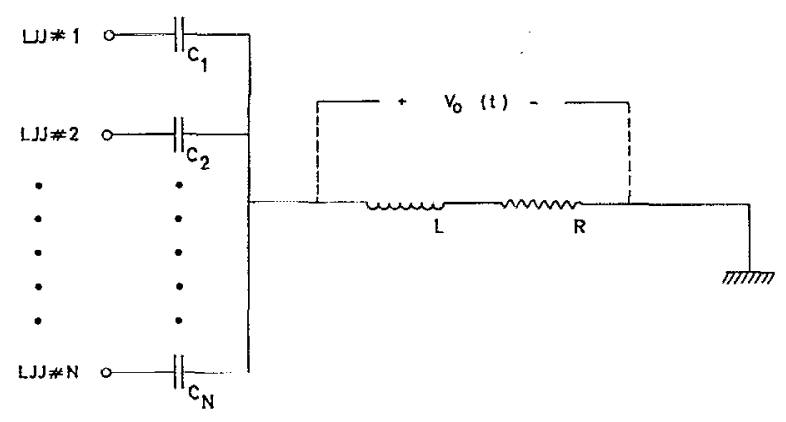

FIG. 1. Simplified model of a junction array coupled to a lumped resonator.

its limitations, we also consider a p.d.e. model of the fluxon dynamics, together with the same lumped resonator, as a "half-way" step towards a complete model, that should contain p.d.e. descriptions of both the junctions and the resonator.

\section{THE MODEL}

The model that we have assumed to describe the array is shown schematically in Fig. 1. Each junction is coupled to the resonator, and thereby to all of the other junctions, through a capacitance; the total effective capacitance of the resonator is just the sum of the individual coupling capacitances. It is clear that this model represents a compromise with physical reality in favor of computational simplicity: physically, we expect the resonator to contain its own fixed capacitance, which is independent of the junction coupling capacitances. This more realistic model, however, contains one more adjustable parameter to worry about; moreover, in light of the results obtained with the model of Fig. 1, we expect its behavior to be fairly readily foreseeable.

Each individual junction in the array is modeled by the standard perturbed sine-Gordon p.d.e., ${ }^{3}$ so that for the $k$ th junction, we have

$$
\varphi_{x x}^{(k)}-\varphi_{t t}^{(k)}-\sin \varphi^{(k)}=\alpha_{k} \varphi_{t}^{(k)}-\beta_{k} \varphi_{x x t}^{(k)}-\gamma_{k},
$$

where the various terms have their usual meanings: $\varphi$ is the phase difference between the junction electrodes, $x$ is the spatial coordinate normalized to the Josephson penetration length $\lambda_{J}, t$ is time normalized to the inverse of the Josephson angular plasma frequency $\omega_{0}$, the term in $\alpha$ represents shunt dissipation caused by quasiparticle tunneling (here, as usual, assumed ohmic), the term in $\beta$ represents dissipation caused by the surface resistance of the junction electrodes, $\gamma$ is the normalized bias current density, and subscripts $x$ and $t$ denote partial derivatives. In the following, we consider only the case $\beta=0$ for simplicity.

We must also specify boundary conditions for Eq. (1) which are determined by the coupling to the resonator. For the general case, i.e., $\beta \neq 0$, these may be expressed $a^{3}$

$$
\begin{aligned}
& \varphi_{x}^{(k)}(0, t)+\beta \varphi_{x t}^{(k)}(0, t)=0 ; \\
& \varphi_{x}^{(k)}\left(L_{k}, t\right)+\beta \varphi_{x t}^{(k)}\left(L_{k}, t\right)=I_{k}(t),
\end{aligned}
$$

where $L_{k}$ is the normalized length of the $k$ th junction, and $I_{k}(t)$ is the current supplied to that junction by the resonator. From Fig. 1 it is readily seen that $I_{k}(t)$ may be calculated as

$$
I_{k}(t)=C_{k}\left[R q_{t t}+\mathscr{L} q_{t t t}-\varphi_{t t}^{(k)}\left(L_{k}, t\right)\right],
$$

where $q(t)$ is the total charge in the resonator $\left[q_{t}(t)\right.$ is the current in the inductance $\mathscr{L}$ of Fig. 1], which in turn is determined by the equation

$$
q_{t t}+2 a q_{t}+\Omega^{2} q=\Omega^{2} \sum_{k=1}^{N} C_{k} \varphi_{t}^{(k)}\left(L_{k}, t\right)
$$

where $a \equiv R / 2 \mathscr{L}, \Omega \equiv\left(\mathscr{L} C_{\text {tot }}\right)^{-1 / 2}, C_{\text {tot }} \equiv \Sigma_{k} C_{k}$ and $N$ is the number of junctions.

Due to manufacturing tolerances, real physical junctions in an array are never identical: we must expect some spread in the values of the parameters $\alpha_{k}, L_{k}$, and $\gamma_{k}$ (the array is assumed to be series-biased, which means that the total physical bias current through each junction is the same; however, $\gamma_{k}$ is a normalized bias current per unit length, depending both upon $L_{k}$ and upon the maximum Josephson current density, to which it is normalized and which may vary slightly from junction to junction). In terms of the normalized current-voltage $(I-V)$ characteristics of the first zero-field step (ZFS) of the junctions in the array, differences in $\gamma_{k}$ are reflected directly as differences in the current-bias point for the various junctions. Differences in $L_{k}$ are reflected in differences in the asymptotic limiting voltage, and hence the asymptotic limiting frequency, of the ZFSs, since the normalized value of this asymptotic voltage is just $2 \pi / L_{k}$. Differences in $\alpha_{k}$ are reflected as differences in the current-voltage profile of the ZFSs along both the current and the voltage axes. Since the basic problem in phase locking is to bring into synchronous frequency (i.e., voltage) operation junctions whose freerunning states are somewhat different, which of these parameter variations is most important in practice will depend upon the current-bias point at which the array is operated. In particular, at relatively low values of the bias current where the dynamic resistance of the ZFSs is relatively large, small variations in $\gamma_{k}$ may give rise to large variations of the free-running voltages (i.e., frequencies), whereas variations in $L_{k}$ will have relatively little signifcance. At relatively high values of the bias current, on the other hand, where the dynamic resistance of the ZFSs is relatively small and all of the junction voltages are close to their respective asymptotic limiting values, variations in $L_{k}$ will be important, whereas variations in $\gamma_{k}$ will not. In the present article, for simplicity, we consider only variations in $L_{k}$, i.e., we assume that $\gamma_{k}=\gamma$ and $\alpha_{k}=\alpha$, for all $k$.

\section{p.d.e. SOLUTION}

The p.d.e. scheme used to simulate the model of Eqs. (1)-(4) is based on an explicit finite-difference method. The p.d.e., Eq. (1), corresponding to each of the $N$ junctions is spatially discretized into $M$ points; in the present work we have used a value of $M \sim 15$ times the normalized length of the junctions. In the interior of each junction we 
use a 5-point approximation to the second spatial derivative, whereas at the two junction ends a 3-point formula is used. ${ }^{5}$ This procedure reduces the model to the following set of $2 M N+2$ first-order ordinary differential equations:

for each junction, at section $m=1$ :

$$
\begin{aligned}
\left(V_{1}\right)_{t}= & \gamma-\alpha V_{1}+2\left(\varphi_{2}-\varphi_{1}\right) /(\Delta x)^{2} \\
& +2 \beta\left(V_{2}-V_{1}\right) /(\Delta x)^{2}-\sin \varphi_{1}, \\
\left(\varphi_{1}\right)_{t}= & V_{1} ;
\end{aligned}
$$

for each junction, at section $m=2$ :

$$
\begin{aligned}
\left(V_{2}\right)_{t}= & \gamma-\alpha V_{2}+\left(\varphi_{3}-2 \varphi_{2}+\varphi_{1}\right) /(\Delta x)^{2} \\
& +\beta\left(V_{3}-2 V_{2}+V_{1}\right) /(\Delta x)^{2}-\sin \varphi_{2}, \\
\left(\varphi_{2}\right)_{t}= & V_{2}
\end{aligned}
$$

for each junction, at section $m, 3<m \leqslant M-2$ :

$$
\begin{aligned}
\left(V_{m}\right)_{t}= & \gamma-\alpha V_{m}+\left(-\varphi_{m+2}+16 \varphi_{m+1}-30 \varphi_{m}\right. \\
& \left.+16 \varphi_{m-1}-\varphi_{m-2}\right) / 12(\Delta x)^{2}+\beta\left(-V_{m+2}\right. \\
& +16 V_{m+1}-30 V_{m}+16 V_{m-1} \\
& \left.-V_{m-2}\right) / 12(\Delta x)^{2}-\sin \varphi_{m} \\
\left(\varphi_{m}\right)_{t}= & V_{m}
\end{aligned}
$$

for each junction, at section $m=M-1$ :

$$
\begin{aligned}
\left(V_{M-1}\right)_{t}= & \gamma-\alpha V_{M-1}+\left(\varphi_{M}-2 \varphi_{M-1}\right. \\
& \left.+\varphi_{M-2}\right) /(\Delta x)^{2}+\beta\left(V_{M}-2 V_{M-1}\right. \\
& \left.+V_{M-2}\right) /(\Delta x)^{2}-\sin \varphi_{M-1}, \\
\left(\varphi_{M-1}\right)_{t}= & V_{M-1} ;
\end{aligned}
$$

for each junction, at section $m=M$ :

$$
\begin{aligned}
\left(V_{M}\right)_{t}= & \gamma-\alpha V_{M}+2\left(\varphi_{M-1}-\varphi_{M}\right) /(\Delta x)^{2}+2 \beta\left(V_{M-1}\right. \\
& \left.-V_{M}\right) /(\Delta x)^{2}-\sin \varphi_{M}+2 I(t) / \Delta x \\
\left(\varphi_{M}\right)_{t}= & V_{M} ;
\end{aligned}
$$

at the common tank node, from Eq. (4):

$$
\begin{aligned}
& q_{t}=p, \\
& p_{t}=-2 a p-\Omega^{2} q+\Omega^{2} \sum_{k=1}^{N} C_{k} V_{M}^{(k)}(t),
\end{aligned}
$$

where $\Delta x$ is the length of a spatial section, given, for the $k$ th junction, by $L_{k} /(M-1)$, and $I(t)$ is the current furnished to the junction by the tank, given, for the kth junction, by Eq. (3). The reduced model of Eqs. (5) is then integrated in time using a routine based on the BulirschStoer algorithm. ${ }^{6}$

The most significant factor contributing to the cost of p.d.e. simulations of this model is the long duration of transients caused by the fact that in order to reasonably model an experimental device ${ }^{1,2}$ it is necessary to employ a fairly large value for the quality factor of the tank $(Q \sim 1000)$. In order to trace the current-voltage charac- teristics of the model with acceptable accuracy, we sweep the bias current $\gamma$ quasiadiabatically, as a linear ramp in time, and we reduce the ramp rate until convergent results are obtained. In practice, typical values of the sweep rate are of the order of $10 \%$ of the critical current for 20000 40000 units of normalized time. The average junction voltage of the $k$ th junction in a stationary state is calculated as $\left\langle V^{(k)}\right\rangle=2 \pi /\left\langle T^{(k)}\right\rangle$; where $T^{(k)}$ is the time of flight for a fluxon to cross the $k$ th junction. Averaging $T^{(k)}$ over $\sim 30$ times of flight yields a voltage uncertainty well below $1 \%$.

\section{PARTICLE-MAP SOLUTION}

The particle-map approach is bascd on the perturbation analysis of fluxon dynamics pioneered by McLaughlin and Scott. ${ }^{7}$ The basic equations describing the behavior of a singe long-junction oscillator in the presence of an external microwave field in the case $\beta=0$ were derived in detail in Ref. 3. This approach was employed, in a slightly different form, in Ref. 1.

In this approach the motion of a fluxon in a junction is reduced to that of a relativistic point particle in a viscous medium; the explicit form of the fluxon trajectory is given in Eq. (13) of Ref. 3. The time of flight $T$ for the fluxon to traverse the junction is found by numerically inverting the trajectory equation. Each time the fluxon in the $k$ th junction reflects from the tank boundary it receives an energy input from the tank, given by ${ }^{8}$

$$
\Delta E_{\text {tank }}^{(k)}=4 \pi I_{k}\left(\tau^{(k)}\right)
$$

where $\tau$ is the time of reflection, and $I_{k}$ is the current defined in Eq. (3). In addition, in order to improve the agreement between the particle-map and the p.d.e. descriptions of fluxon dynamics, we have incorporated into the map an energy loss correction, ${ }^{3}$ similar to that proposed by Pedersen et al. ${ }^{9}$ Each time the fluxon in the kth junction reflects from either the $x=0$ or the $x=L_{k}$ boundary it experiences an energy loss, due to increased dissipation during the reflection, given by

$$
\Delta E_{\text {loss }}^{(k)}=-\pi^{2} \alpha\left[1+4\left(1-u^{(k)}\right) / \pi^{2}\right],
$$

where $u$ is the fluxon velocity at the instant just prior to the reflection. The original correction proposed by Pedersen et $a l^{9}{ }^{9}$ was just twice that given by Eq. (7). Olsen $e t a l .{ }^{10}$ showed that the original correction, together with a correction of opposite sign due to phase-shift effects, notably improves the agreement between particlc-map and p.d.e. descriptions of fluxon dynamics. We have discovered empirically that halving the energy-loss correction and ignoring the phase-shift correction yields an improvement that is almost as good; this procedure has the advantage of being computationally simpler.

In order to complete the particle-map model we must calculate the effect of the junctions on the tank. To do this we assume, consistently with the assumed particle nature of the fluxons, that each fluxon reflecting at the tank boundary can be described as a delta function voltage pulse of weight $4 \pi$. With $N$ junctions in the array, we calculate the effect of one such reflection, in the $k$ th junction, by 
applying a voltage input $4 \pi \delta(t)$ to capacitor $C_{k}$, with the other $N-1$ capacitors grounded. Elementary circuit calculations give the resulting resonator voltage as

$$
\begin{aligned}
V_{0}(t)= & 4 \pi \delta(t) C_{k} / C_{\mathrm{tot}}+[A \cos (\omega t)+B \sin (\omega t)] \\
& \times \exp (-a t),
\end{aligned}
$$

where $A \equiv V_{0}\left(0^{-}\right), \quad B \equiv\left[a V_{0}\left(0^{-}\right)+\dot{V}_{0}\left(0^{-}\right)-4 \pi\left(\omega^{2}\right.\right.$ $\left.\left.+a^{2}\right) C_{k} / C_{\mathrm{tot}}\right] / \omega, \omega \equiv\left(\Omega^{2}-a^{2}\right)^{1 / 2}, V_{0}\left(0^{-}\right)$and $\dot{V}_{0}\left(0^{-}\right)$ are, respectively, the resonator voltage and its time derivative just before the reflection, due to previous reflections, and $a, \Omega$, and $C_{\text {tot }}$ are as defined in Eq. (4). In terms of previously defined quantities we have, evidently, that

$$
V_{0}(t)=R q_{t}(t)+\mathscr{L} q_{t t}(t) .
$$

In what follows we will ignore the delta function term in Eq. (8), and we will assume that all of the coupling capacitors are identical, so that $C_{k} / C_{\text {tot }}=1 / N$. We also note that, due to the high quality factor assumed for the tank $(Q \sim 1000)$, the difference between the frequencies $\omega$ and $\Omega$ is negligible.

We choose, for convenience of calculation, a shifted time scale such that each fluxon reflection in each junction occurs at $t=0$. This means that the energy input from the tank to the fluxon in the $k$ th junction, given by Eq. (6), may be written as

$$
\Delta E_{\text {tank }}^{(k)}=4 \pi C_{k} \dot{V}_{0}\left(0^{+}\right)=4 \pi C_{k}(\omega B-a A) .
$$

The computational procedure for calculating the dynamics of the system may thus be schematized as follows: (1) Choose arbitrary initial values for $V_{0}(0)$ and $\dot{V}_{0}(0)$; these determine initial values for the constants $A$ and $B$. (2) Launch a fluxon in each junction from the $x=0$ end with some initial velocity. (3) When the first fluxon, in for example, the $k$ th junction, reaches the resonator boundary at, for example, time $t=\tau^{(k)}$, determined by inverting the trajectory equation, calculate $V_{0}\left(\tau^{(k)}\right)$ and $\dot{V}_{0}\left(\tau^{(k)}\right)$ from $\mathrm{Eq}$. (8). (4) Shift the time scale so that $V_{0}\left(\tau^{(k)}\right)=V_{0}\left(0^{-}\right)$ and $\dot{V}_{0}\left(\tau^{(k)}\right)=\dot{V}_{0}\left(0^{-}\right)$; from these new initial conditions calculate new values for the constants $A$ and $B$. (5) Calculate the energy increment of the fluxon in the $k$ th junction from Eq. (10) [also taking into account Eq. (7) for the energy loss during reflection]. (6) Iterate until the system converges to a steady state.

If the resulting steady state is a phase-locked state the average time of flight of a fluxon across a junction will assume the same constant value, $T_{\text {avy }}$, for all of the active junctions in the array [we note that the average must be taken over an even number of times since the left-to-right time is, in general, different from the right-to-left time because of the asymmetry of the boundary conditions of Eqs. (2)]. In this situation the average normalized junction voltage for each of the junctions will be $V_{\mathrm{av}}=2 \pi / T_{\mathrm{av}}$.

In principle, the computational procedure outlined above is formally equivalent to an $N+2$ dimensional functional map; the map variables may be taken to be the constants $A$ and $B$ plus the average times of flight $T_{\mathrm{ay}}^{(k)}, k$ $=1,2, \ldots, N$. In this context, a phase-locked state of the system dynamics corresponds to a fixed point of the map,

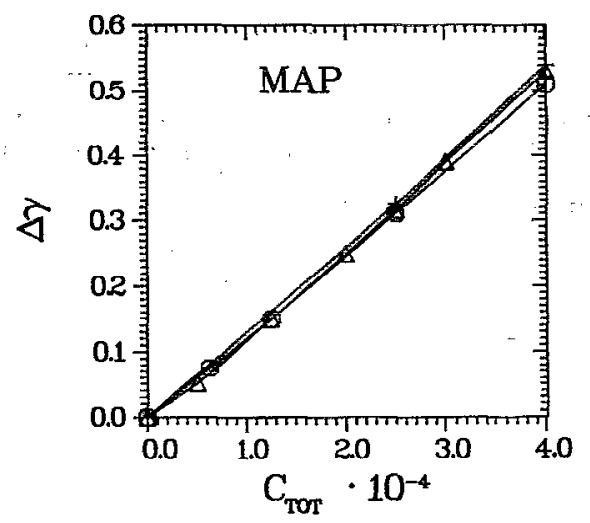

FIG. 2. Dependence of locking range in bias current on total tank capacitance using the particle-map approach. Circles: $N=1$ junction with $L_{1}$ $=3.00$; triangles: $N=2$ junctions with $L_{1}=3.00, L_{2}=2.94$; crosses: $N=4$ junctions with $L_{1}=3.00, L_{3}=2.98, L_{3}=2.96, L_{4}=2.94$. Solid lines are intended only as a guide to the eye. Junction loss parameter $\alpha_{k}=0.05$ for all junctions. Tank parameters: $\omega=0.97, Q \approx \omega / 2 a=1000$.

determined by imposing $A_{n+1}=A_{n}, \quad B_{n+1}=B_{n}$, and $\left(T_{\mathrm{av}}^{(k)}\right)_{n+1}=\left(T_{\mathrm{av}}^{(k)}\right)_{n}=T_{\mathrm{av}}$, for all $k$, where $n$ is the iteration number, comprising one cycle through all $N$ junctions. Such a fixed point exists for a certain range, $\Delta \gamma$, of the junction bias current. It is manifested as a nearly constantvoltage step in the $I-V$ characteristics of the first ZFS of the junctions in the array, at a voltage corresponding to the resonant frequency of the resonator ( $V=2 \omega$ with our normalizations). In the locking region, obviously, the characteristics of all of the junctions in the array coincide (see, e.g., Fig. 5 of Ref. 1).

\section{NUMERICAL RESULTS}

Figure 2 shows the height in bias current, $\Delta \gamma$, of the phase-locking regions obtained using the particle-map approach, as a function of the total tank capacitance, $C_{\mathrm{tot}}$, for $N=1,2$, and 4 junctions. The total spread in junction lengths for $N=2$ and $N=4$ was taken as $2 \%$. All three dependences are essentially proportional to $C_{\text {tot }}$, and all three have essentially the same slope. These facts can be explained qualitatively using a simple argument developed in Ref. 1: With a single junction in a steady state, $V_{0}\left(0^{+}\right)$ can be obtained explicitly from Eq. (8) by summing impulse responses for a semi-infinite train of periodic voltage impulses, and the current $I_{1}$ flowing into the junction from the resonator at $t=0^{+}$is obtained by summing the time derivatives of these impulse responses. This latter sum is readily shown to be proportional to $C_{1}$, which in this case is the same as $C_{\text {tot }}$ [see Eq. (3) of Ref. 1]. Specifically, for a high- $Q$ resonator we find to good approximation that

$$
I_{1} \approx 4 Q \omega^{2} C_{1} \text {, }
$$

where $Q \approx \omega / 2 a$ is the quality factor. Moreover, the height of the bias current step is given approximately by $\Delta \gamma_{1} \approx I_{1} /$ $L_{1}$ [see Eq. (5) of Ref. 1], which is thus also proportional to $C_{\text {tot }}$. For two junctions, with $C_{1}=C_{2}=C_{\text {tot }} / 2$, the current into each junction would be approximately halved for the same $C_{\mathrm{tot}}$, but $V_{0}\left(0^{+}\right)$and $\dot{V}_{0}\left(0^{+}\right)$are approximately 


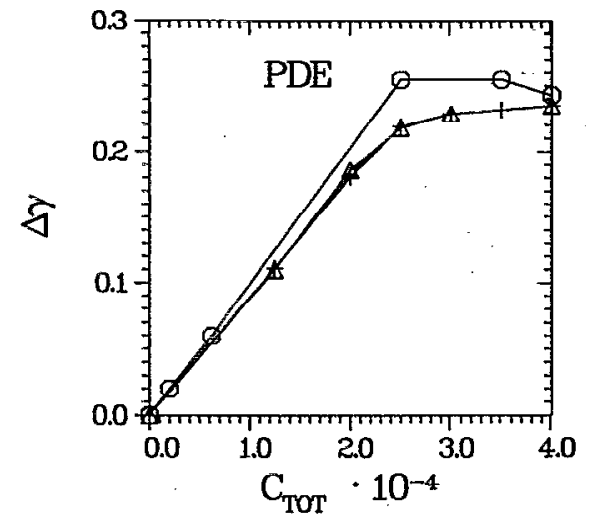

FIG. 3. Same as Fig. 2, but using the p.d.e. approach.

doubled, since there are now two junctions resonantly pumping the tank, so the bias current step is approximately unchanged. Accordingly, we may in general write that

$$
\Delta \gamma \approx 4 Q \omega^{2} C_{\text {tot }} / L_{\text {av }}
$$

where $L_{\mathrm{av}}$ is the average junction length.

In Fig. 3 we show the corresponding result for the p.d.e. approach. Two salient differences with respect to Fig. 2 are apparent: (1) Figure 3 shows a saturation region for higher values of $C_{\text {tot }}$ that is not present in Fig. 2; (2) Before the saturation region, for a given value of $C_{\text {tot }}$, the p.d.e. value of $\Delta \gamma$ is $\sim 30 \%$ smaller than the particle-map value. The latter difference, i.e., the fact that the p.d.e. step is smaller than the particle-map step, is probably attributable to the fact that a fluxon in the particle-map formalism is a point particle with no spatial extension, whereas a p.d.e. fluxon has a non-zero width. Consequently, whereas energy exchange at the tank boundary occurs instantaneously in the map, the exchange interaction is smeared over a certain time interval in the p.d.e. This difference will tend to disappear for junction lengths $L_{k}>1$, but it is significant for the values used in the present work $\left(L_{k} \sim 3\right)$.

The appearance of the saturation region in Fig. 3 is attributable to the fact that for increasing values of $C_{\text {tot }}$ and hence for increasing values of $I_{k}\left(\tau^{(k)}\right)$, a dynamic mechanism not contained in the particle-map formalism begins to appear, viz., the creation of additional fluxons in the junction (the particle-map formalism assumes that the number of fluxons in each junction is always one; fluxon creation is not allowed). This mechanism has been noted in previous comparisons of p.d.e. and particle-map results (see, e.g., Fig. 15 of Ref. 4), and it is further borne out by the results shown in Fig. 4, which depicts the junction $I-V$ characteristics for the case of $N=4$ junctions, for two different values of $C_{\text {tot. }}$. The solid curves in Fig. 4 were obtained by integration of the p.d.e. model; the dotted curves are the unperturbed (i.e., no tank) ZFS profiles, obtained using the particle-map approach. In both Figs. 4 (a) and 4 (b) we seen a current step at $V \approx 1.94$, where all four junctions are locked to the tank. As the bias current $\gamma$ is raised, the junctions unlock one by one; the first junction to switch is the shortest in the array, followed by the other progres-
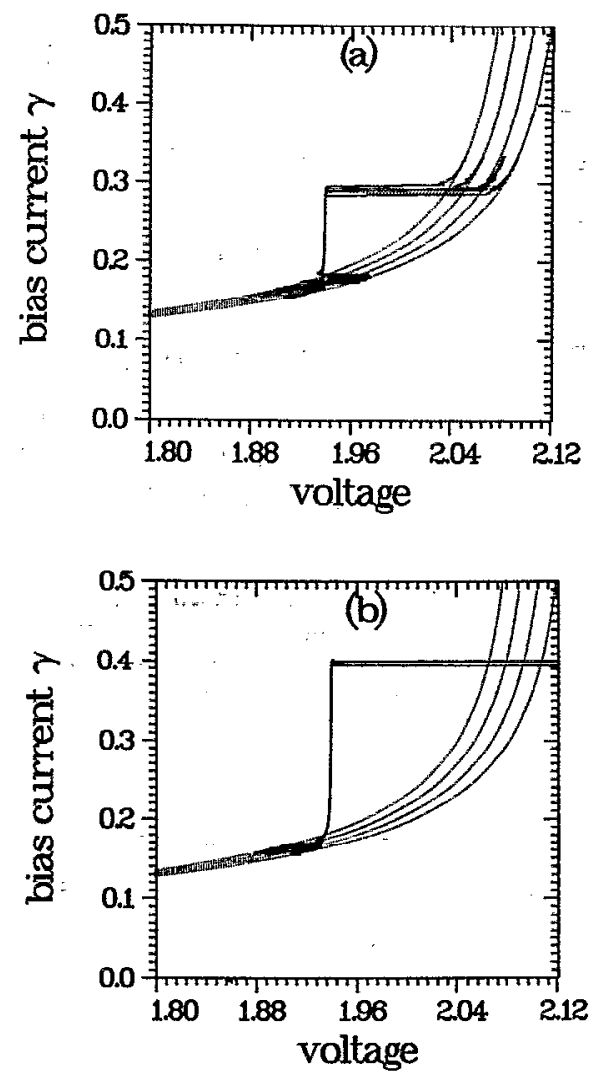

FIG. 4. Junction current-voltage characteristics near the locking region for $N=4$, obtained using the p.d.e. approach (solid curves), for (a) $C_{\text {tot }}$ $=1.25 \times 10^{-4}$, (b) $C_{\text {tot }}=2.50 \times 10^{-4}$. The dotted curves are the unperturbed characteristics (i.e., no tank), obtained using the particle-map approach. Junction parameters: $L_{1}=3.00, L_{2}=2.98, L_{3}=2.96, L_{4}=2.94$, $\alpha_{k}=0.05$ for all junctions. Tank parameters: $\Omega=0.97, Q \approx \Omega / 2 a=1000$.

sively longer junctions. In Fig. 4(a), for which $C_{\text {tot }}$ is below the saturation region shown in Fig. 3, we see that the switch takes each junction from the top of the step to the unperturbed ZFS1, i.e., the dynamic configuration remains that of one fluxon in each junction. In Fig. 4(b), on the other hand, where $C_{\text {tot }}$ is within the saturation region shown in Fig. 3, the switch clearly takes the junctions to some higher-order state, i.e., additional fluxons have entered the junctions. We note parenthetically that the fact that the solid and dotted curves in Fig. 4(a) coincide outside of the locking region gives an indication of the effectiveness of the energy-loss correction of $\mathrm{Eq}$. (7) in the particle-map approach.

Within the locking region the relative phase of the fluxon reflection in each junction assumes a specific value with respect to the phases of the other junctions; each junction adjusts its phase with respect to the resonator voltage (or current) in such a way that it receives from the resonator the energy increment which, in general, may be either positive or negative, needed to offset its frequency from the free-running value to the phase-locked value. This fact is illustrated in detail in Fig. 5 , which is a p.d.e. result corresponding to Fig. 4(a), To construct Fig. 5 we define $i_{\max } \equiv\left(q_{t}\right)_{\max }$, i.e., the maximum value of resonator current, and we define $i_{\text {ref }}^{(k)} \equiv q_{t}\left(\tau^{(k)}\right)$, i.e., the value of this current at the instant that the fluxon in the $k$ th junction 


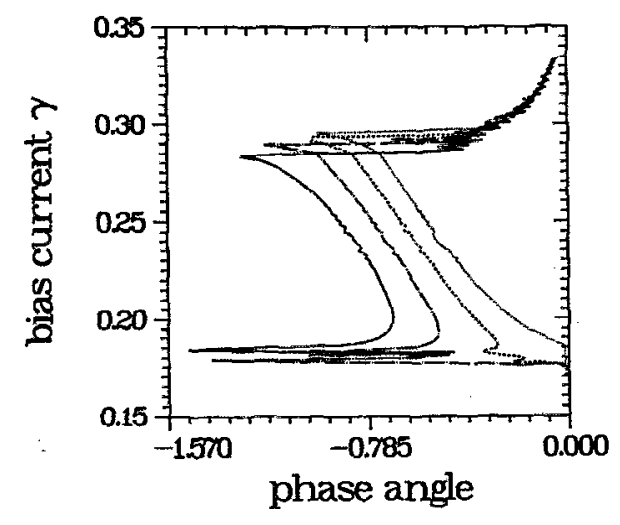

FIG. 5. Relative junction phase angles, defined in Eq. (13) in the text, within the locking region, corresponding to Fig. 4(a), obtained using the p.d.e. approach. Proceeding from right to left, the junction lengths (those of Fig. 4) corresponding to the four curves become progressively smaller.

refiects from the tank boundary. Since the resonator current is very nearly sinusoidal, we calculate the phases simply as

$$
\vartheta_{k}=\arcsin \left(i_{\mathrm{ref}}^{(k)} / i_{\max }\right)
$$

A priori, the phase angles $\vartheta_{k}$ can vary in the range $-\pi /$ $2<\vartheta_{k}<+\pi / 2$. To understand the result shown in Fig. 5 we observe that, because of the high $Q$-factor, the tank voltage is very nearly the same as the voltage across the inductance $\mathscr{L}$ in Fig. 1 , i.e., $V_{0}(t)$ is very nearly proportional to the time derivative of the tank current. The current $I_{k}(t)$ furnished by the tank to each junction is proportional to $\dot{V}_{0}(t)$, i.e., proportional to the second derivative of the tank current. Since the tank voltage and current are very nearly sinusoidal, this means that $I_{k}(t)$ is proportional to the negative of the tank current. A positive current into a junction adds energy to a fluxon reflecting at one boundary and subtracts energy at the other boundary; ${ }^{3}$ with the reference polarities that we have chosen, the tank boundary is a subtracting boundary. As is evident from Fig. 4, the effect of the tank is to shift the junction $I-V$ characteristics to lower voltage values, i.e., lower energies, with respect to the free-running values. Accordingly, we expect to find $-\pi / 2 \leqslant \vartheta_{k}<0$, which is just the situation shown in Fig. 5.

In Fig. 6 we show in further detail the dynamics of the locking procedure for the case of $N=2$ junctions, obtained via p.d.e. simulation; in this figure the solid and dotted curves are, respectively, the junction voltage peaks for the longer and the shorter junctions at the tank boundary, and the dashed curve is the tank current. In Fig. 6(a) the junction bias current $\gamma$ is slightly below the locking region; we see clearly that the phase relationships vary with time, indicating that locking of both junctions has not yet occurred. In Fig. 6(b) the current $\gamma$ has been increased; here we see that all of the wave forms are strictly periodic in time, with a fixed phase relationship. We note also that, with two junctions locked to the tank, the tank current amplitude approximately doubles.
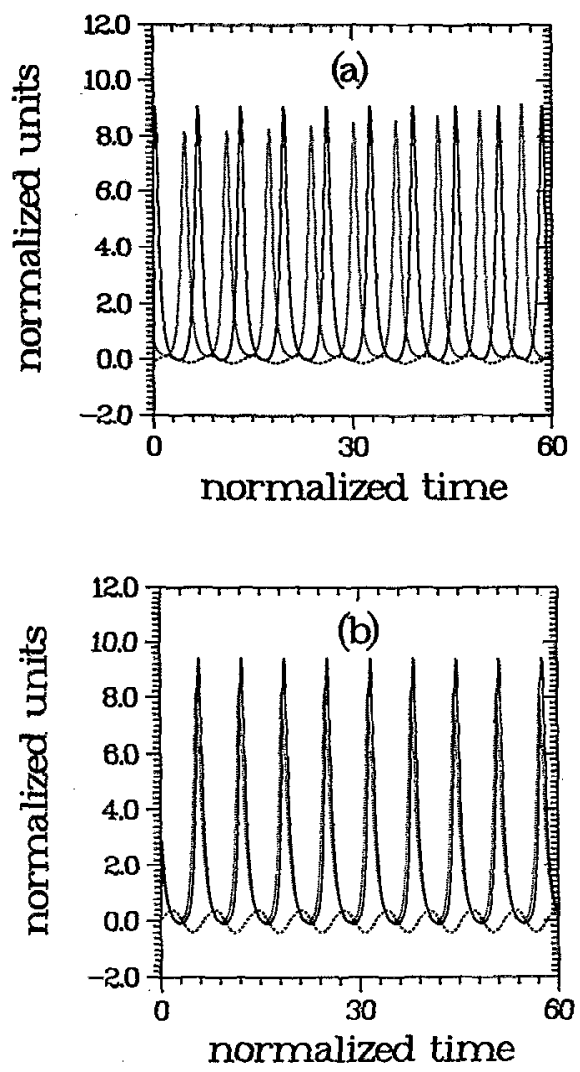

FIG. 6. Dynamics of the locking process for $N=2$ junctions, obtained using the p.d.e. approach. Solid curve: junction voltage of the longer junction at the tank boundary; dotted curve: junction voltage of the shorter junction at the tank boundary; dashed curve: tank current. (a) $\gamma=0.190$ (below the locking region); (b) $\gamma=0.253$ (within the locking region). Junction parameters: $L_{1}=3.00, L_{2}=2.94, \alpha_{1}=\alpha_{2}=0.05$. Tank parameters: $\Omega=0.97, Q=1000$.

\section{MINIMUM CAPACITANCE FOR LOCKING}

The existence of a minimum value of the coupling capacitance, or equivalently a maximum value of the resonator frequency to obtain locking of two junctions, may be established using the following simple, intuitive argument: In order to lock at a given frequency (i.e., voltage $V=2 \omega$ ) two junctions having different lengths, it is necessary that the current height of the locking step for one junction, i.e., one-half the value given by $\mathrm{Eq}$. (12), be at least as large as the separation in current between the free-running $I-V$ characteristics of the two junctions at that voltage. In other words, the shorter junction, working alone, must be able to "touch" the longer one. In terms of the voltage, this means from Eq. (12), that

$$
Q V^{2} C_{\text {tot }} / 2 L_{\text {av }} \geqslant \gamma_{1}(V)-\gamma_{2}(V) \text {. }
$$

Figure 7 shows separately the two sides of relation (14) for three different values of $C_{\text {tot }}$ : the three solid curves are the left-hand side and the dotted curve is the righthand side for a particular choice of system parameters. To calculate this latter term, we have simply iterated numerically the particle-map in the absence of the tank to obtain $\gamma_{1}(V)$ and $\gamma_{2}(V)$; these are slightly different from the usual power-balance expressions ${ }^{1,3}$ since we have incorpo- 


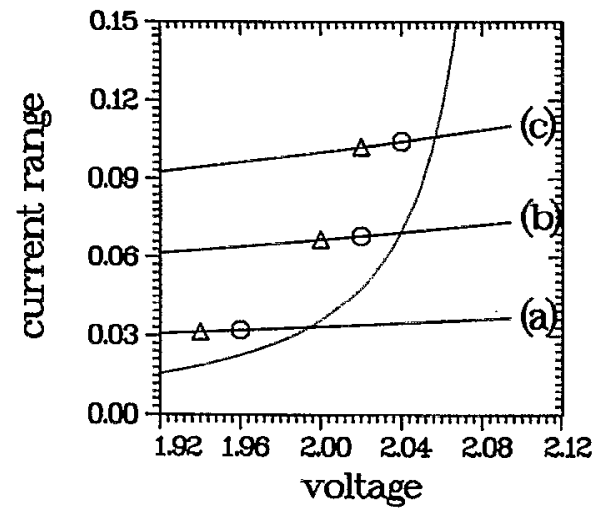

FIG. 7. Minimum capacitance/maximum voltage for locking two junctions. Dotted curve: right-hand side of (14); solid curves: left-hand side of (14) for (a) $C_{\text {tot }}=0.50 \times 10^{-4}$, (b) $C_{\text {tot }}=1.00 \times 10^{-4}$, (c) $C_{\text {tot }}=1.50$ $\times 10^{-4}$. Relation (14) predicts locking only to left of intersection points; triangles and circles define limits obtained by map iteration. Parameters: $L_{1}=3.00, L_{2}=2.94, \alpha_{1}=\alpha_{2}=0.05, Q=1000$.

rated the energy loss correction of Eq. (7). In Fig. 7 the points of intersection between the dotted and solid curves are the upper-voltage limits for locking according to relation (14); the intervals between the triangle and the circle on each of the three solid curves are, instead, the corresponding limits obtained by direct numerical iteration of the complete particle-map (using a frequency increment of 0.01 ). The agreement seems quite reasonable, particularly in view of the rough, approximate nature of relation (14). Moreover, preliminary results obtained via p.d.e. simulation are qualitatively consistent, and indeed in approximately quantitative agreement, with the particle-map results shown in Fig. 7.

Presumably, a qualitatively similar argument may be applied in the case of more than two junctions. This might well give rise to a mechanism that limits the total number of junctions that can be locked together in a given experimental array.

\section{CONCLUSIONS}

Phase-locked arrays of long Josephson junction fluxon oscillators, operating in the resonant propagation mode, show considerable promise for practical applications in, for example, local oscillators for SIS (superconductorinsulator-superconductor) mixers. Single-junction oscillators of this type offered the attractive feature of a very narrow output linewidth, but they seemed unable to furnish enough output power to be practically useful. Accordingly, attention for practical applications shifted to the flux-flow type oscillator pioneered by Nagatsuma et al. ${ }^{11}$ The stratagem of using arrays of resonant oscillators, however, might well overcome the shortcoming of low output power while maintaining a narrow linewidth.

The p.d.e. models needed to describe such devices have steadily become more costly in terms of computer time as the devices have become more complicated. The particlemap approach yields a surprisingly detailed agreement with full simulations of the p.d.e. models, with a substantial saving-typically three orders of magnitude-in computer time. It thus offers a computationally powerful and economical tool for initial exploration of the design features of such arrays. A specific problem of practical interest that may be affronted conveniently using this approach is the total number of junctions in a given array that may be locked together as a function of the array parameters.

\section{ACKNOWLEDGMENTS}

Three of us (GF, GR, RDP) wish to thank the MIDIT Center of the Technical University of Denmark, where much of this work was performed, for the warm hospitality received. We gratefully acknowledge financial support from MIDIT (Denmark) and from MURST, the Fondazione Angelo Della Riccia, and the Progetto Finalizzato "Tecnologie Superconduttive e Criogeniche" del CNR (Italy).

${ }^{1}$ R. Monaco, N. Gronbech-Jensen, and R. D. Parmentier, Phys. Lett. A 151, 195 (1990).

${ }^{2}$ A. Davidson, N. Grønbech-Jensen, and N. F. Pedersen, IEEE Trans. Magn. 27, 3347 (1991); see also A. Davidson and N. F. Pedersen, Appl. Phys. Lett. 60, 2017 (1992).

${ }^{3}$ M. Salerno, M. R. Samuelsen, G. Filatrella, S. Pagano, and R. D. Parmentier, Phys. Rev. B 41, 6641 (1990).

${ }^{4}$ N. F. Pcdersen and A. Davidson, Phys. Rev. B 41, 178 (1990).

${ }^{5} \mathrm{M}$. Abramowitz and I. Stegun, Handbook of Mathematical Functions, 9th ed. (Dover, New York, 1970), chap. 25.

${ }^{6}$ W. H. Press, B. P. Flannery, S. A. Teukolsky, and W. T. Vetterling, Numerical Recipes (Cambridge University Press, Cambridge, 1986), chap. 15.

${ }^{7}$ D. W. McLaughlin and A. C. Scott, Phys, Rev. A. 18, 1652 (1978).

${ }^{8}$ O. A. Levring, N. F. Pedersen, and M. R. Samuelsen, J. Appl. Phys. 54, 987 (1983).

${ }^{9}$ N. F. Pedersen, M. R. Samuelsen, and D. Welner, Phys. Rev. B 30, 4057 (1984).

${ }^{10}$ O. H. Olsen, N. F. Pedersen, M. R. Samuelsen, H. Svensmark, and D. Welner, Phys. Rev. B 33, 168 (1986).

${ }^{11}$ T. Nagatsuma, K. Enpuku, F. Irie, and K. Yoshida, J. Appl. Phys. 54, 3302 (1983). 